\title{
Dissipation and supercurrent fluctuations in a diffusive NS ring
}

\author{
B. Dassonneville ${ }^{1}$, M. Ferrier ${ }^{1}$, S. Guéron ${ }^{1}$ and H.Bouchiat ${ }^{1}$ \\ 1 LPS, Univ. Paris-Sud, CNRS, UMR 8502, F-91405 Orsay Cedex, France
}

\begin{abstract}
A mesoscopic hybrid Normal/Superconducting (NS) ring is characterized by a dense Andreev spectrum with a flux dependent minigap. To probe the dynamics of such a ring we measure its linear response to a high frequency flux, in a wide frequency range, with a multimode superconducting resonator. We find that the current response contains, beside the well known dissipationless Josephson contribution, a large dissipative component. At high frequency compared to the minigap and low temperature we find that the dissipation is due to transitions across the minigap. In contrast, at lower frequency there is a range of temperature for which dissipation is caused predominantly by the relaxation of the Andreev states' population. This dissipative response, related via the fluctuation dissipation theorem to a non intuitive zero frequency thermal noise of supercurrent, is characterized by a phase dependence dominated by its second harmonic, as predicted long ago [1, 2] but never observed so far.
\end{abstract}

PACS numbers:

A phase coherent non superconducting conductor $(\mathrm{N})$ connected to two superconductors (an SNS junction) gives rise to the formation of Andreev states (AS) which are coherent superpositions of electron and hole states confined in the $\mathrm{N}$ metal and carry the Josephsonsupercurrent 3]. They strongly depend on the phase difference $\varphi$ between the two superconductors. The quasi-continuous Andreev spectrum of a diffusive metallic wire exhibits a phase modulated induced gap, the minigap, which closes at odd multiples of $\pi$ (for perfectly transmitting contacts), and can be approximated by $2 E_{g}(\varphi)=2 E_{g}(0)|\cos \varphi / 2|$ [4]. In the case of a wire longer than the superconducting coherence length, $E_{g}(0) \simeq 3 E_{T h}$, with $E_{T h}$ the Thouless energy $\hbar / \tau_{D}$, and $\tau_{D}$ the diffusion time across the $\mathrm{N}$ wire. Whereas most investigations of diffusive SNS junctions rely on non linear transport measurements of current voltage curves, switching current and ac Josephson effect [7, 8, only few experiments probe the Andreev spectrum at equilibrium in a phase biased configuration with NS rings threaded by an Aharonov Bohm flux. These include the tunnel spectroscopy of the minigap [9] and the measurement of the flux dependent Josephson supercurrent using SQUID 10 or Hall probe magnetometry 11. Beyond probing the equilibrium AS spectrum in a static magnetic flux, the investigation of the dynamics associated to this spectrum, a far more complex question, has been addressed experimentally only recently 12$]$. Theoretically, it was predicted that, in contrast to tunnel Josephson junctions [13] and because of the smallness of the induced gap, SNS junctions should exhibit low frequency supercurrent fluctuations at equilibrium 2. According to the fluctuation dissipation theorem, in the linear response regime, such equilibrium fluctuations lead to a dissipative current under an ac flux excitation 14, 15. In this Letter, we present the linear current response of a phase biased NS ring and account for both the non dissipative component and the more surprising dissipative one, over a wide frequency range. We identify two fundamental processes leading to dissipation, the microwave-induced transitions across the minigap, and the energy relaxation of Andreev level populations.

To this end, we couple a NS ring to a superconducting resonator, and phase bias it with a dc Aharonov Bohm flux and a small ac flux $\delta \Phi_{\omega}$ at the resonator's eigenfrequencies $\omega$. The linear current response $\delta I_{\omega}$ is characterized by the complex susceptibility $\chi(\omega)=\delta I_{\omega} / \delta \Phi_{\omega}=$ $\chi^{\prime}(\omega)+i \chi^{\prime \prime}(\omega)=i \omega Y(\omega)$, where $Y(\omega)$ is the NS ring's admittance. This susceptibility is extracted from the variations of the resonator's eigenmodes (frequency and quality factor). The ring's dissipationless response is deduced from the periodic flux variations of $\chi^{\prime}$, whereas the dissipation corresponds to $\chi^{\prime \prime}$.

A first experiment [12] found a large dissipative response as well as a non dissipative one that differed notably from the adiabatic susceptibility, the simple flux derivative of the ring's Josephson current (the inverse kinetic inductance). These results were partially explained by the theory of the proximity effect 15 . However, the shape of the flux dependences of $\chi$ did not vary with frequency (in the range explored), so that the different components of the ring's dynamical response could not be accessed. In particular, with the inelastic scattering rate much smaller than the lowest eigen-frequency, the dissipative response associated to the relaxation of Andreev states could not be detected.

In the present experiment, we report on a NS ring with enhanced temperature dependent inelastic scattering rate $1 / \tau_{i n}(T)$, thanks to a thin Pd layer at the NS interface, between the normal gold mesoscopic wire and the superconducting niobium loop. The higher inelastic scattering rate, combined to a broader frequency and temperature range, lead to the identification of the two fundamental contributions to the supercurrent relaxation. At frequencies above the inelastic scattering rate, dissipation is due to microwave-induced excitations across the mini- 


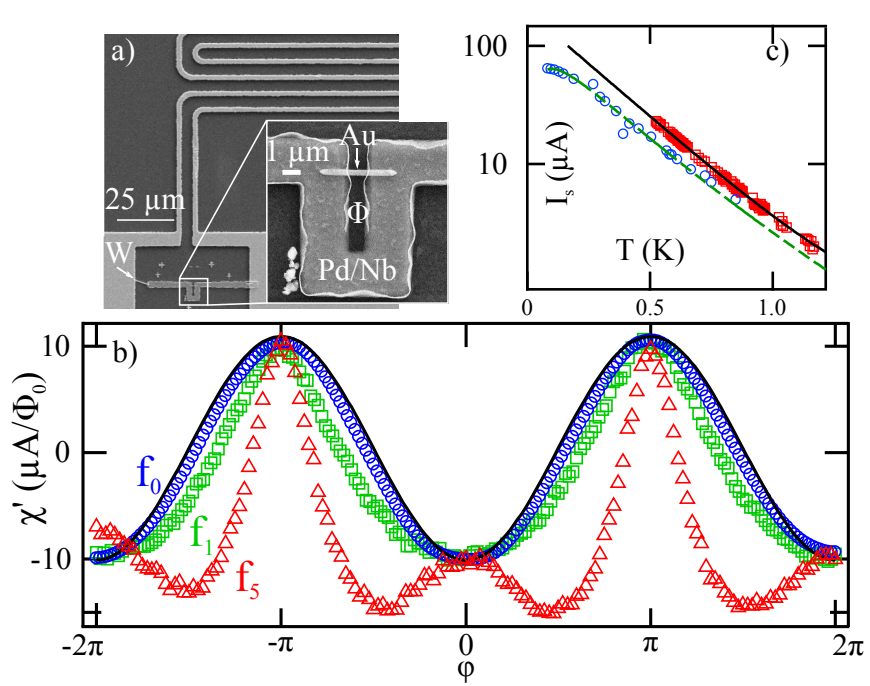

FIG. 1: a. Inset : Scanning electron micrograph of the NS ring fabricated to explore the dynamics of Andreev States. a.NS ring is connected to a $\mathrm{Nb}$ multimode resonator (meander in the top) by W wires deposited by a FIB. b. Flux dependence of $\chi^{\prime}$ at several resonator eigenfrequencies $\left(f_{0}=190 \mathrm{MHz}, f_{1}=560 \mathrm{MHz}, f_{5}=2 \mathrm{GHz}\right)$ and $\mathrm{T}=1.2 \mathrm{~K}$. At $f_{0}, \chi^{\prime}$ is barely distinguishable from a cosine (solid line). Whereas the amplitude $\delta \chi_{\pi-0}^{\prime}=\chi^{\prime}(\pi)-\chi^{\prime}(0)$ is frequency independent, its temperature dependence is that of the switching current $I_{s}$. Note the appearance of a local maximum around $\varphi=0$ at high frequency. c. Temperature dependence of the switching current of the control sample $I_{s}$ (circles) and of $\frac{\Phi_{0}}{2 \pi} \frac{\mathcal{L}}{L_{C}^{2}} \delta \chi_{\pi-0}^{\prime}$ measured in the NS ring (squares) with their fits according to ref. [8] (respectively dashed and solid lines). Best fit yields $E_{T h} \equiv 71 \mathrm{mK} \pm 5 \mathrm{mK}$ corresponding to $1.5 \mathrm{GHz}$ for the NS ring.

gap. In the opposite regime of lower frequency, which could not be reached previously, dissipation is due to the relaxation of Andreev level populations.

Here we reveal this second contribution, proportional to the sum of the squared Andreev level currents. Accordingly we measure a response whose period in flux is nearly $\pi$ periodic. The extra cusps we find at odd multiples of $\pi$ reflects the closing of the minigap. This characteristic phase dependence, which is precisely that of the low frequency, thermal supercurrent noise, is in complete agreement with theoretical predictions formulated long ago [1, 2, 15].

The experimental set-up is shown in Fig.1a, the resonator consists in a double meander line etched out of a 1 micron thick niobium film sputtered onto a sapphire substrate. The NS ring connects the two lines at one end of the resonator, turning it into a $\lambda / 4$ line with a fundamental frequency of $190 \mathrm{MHz}$, and harmonics $380 \mathrm{MHz}$ apart. A weak capacitive coupling to the microwave generator preserves the high quality factor of the resonances, which can reach $510^{4}$ up to $10 \mathrm{GHz}$. The NS ring is fabricated by electron beam lithography. The Au wire (4 micron long, 0.3 micron wide and $50 \mathrm{~nm}$ thick) is first deposited by e-beam deposition of high purity gold. The S part is deposited in a second alignment step by sputtering of a $\mathrm{Pd} / \mathrm{Nb}$ bilayer (6 nm $\mathrm{Pd}, 100 \mathrm{~nm} \mathrm{Nb}$ ). The resulting uncovered length of the $\mathrm{Au}$ wire is $1 \mu \mathrm{m}$. The ring is connected to the $\mathrm{Nb}$ resonator in a subsequent step, using ion-beam assisted deposition of a tungsten wire in a focused ion beam (FIB) microscope. This process creates a good superconducting contact between the resonator and the $\mathrm{Pd} / \mathrm{Nb}$ part of the ring. The $6 \mathrm{~nm}$-thick $\mathrm{Pd}$ buffer layer ensures a good transparency at the NS interface, as demonstrated by the amplitude of the critical current measured with dc transport measurements on control SNS junctions fabricated simultaneously (Fig.1c). It also enhances the inelastic scattering rate because of Pd's spin-wave like excitations (paramagnons) [16 18. Considering that the phase coherence time extracted from weak localization measurements on a $6 \mathrm{~nm}$ thick $\mathrm{Pd}$ thin film [16], was of the order of $0.3 \pm 0.1 \mathrm{~ns}$ at $1 \mathrm{~K}$, which is longer than the estimated diffusion time $\tau_{D}=0.1 \mathrm{~ns}$ through the Au wire between the S contacts, we do not expect a reduction of the critical current as confirmed by measurements in the control samples.

The quantities we measure are the variations with $\mathrm{dc}$ flux of the resonator's quality factor and eigenfrequencies $\delta Q(\Phi)$ and $\delta f(\Phi)$. They are simply related to the oscillating phase dependent part of the complex susceptibility, characterized by $\chi^{\prime}(\varphi)$ and $\chi^{\prime \prime}(\varphi)$, where the superconducting phase $\varphi$ is related to the flux threading the ring by $\varphi=-2 \pi \Phi / \Phi_{0}$ where $\Phi_{0}=h / 2 e$ is the superconducting flux quantum. The relation reads [12]:

$$
\frac{\delta f_{n}(\Phi)}{f_{n}}=-\frac{1}{2} \frac{L_{C}^{2}}{\mathcal{L}} \chi^{\prime}(\varphi), \delta \frac{1}{Q_{n}}(\Phi)=\frac{L_{C}^{2}}{\mathcal{L}} \chi^{\prime \prime}(\varphi) .
$$

The coupling inductance $L_{C}=9 \pm 2 p H$ is due to the $\mathrm{S}$ part of the $\mathrm{N} / \mathrm{S}$ ring; $\mathcal{L}=0.3 \mu H$ is the inductance of the resonator. These expressions are valid at temperatures such that the kinetic inductance of the SNS junction is larger than the ring's geometrical inductance (outside this range screening of the applied flux, both dc and ac, needs to be considered [12]). This sets the lower limit to the temperature, so that experiments were conducted between 0.4 and $1.5 \mathrm{~K}$. The frequencies probed ranged between $190 \mathrm{MHz}$ and $3 \mathrm{GHz}$.

We find spectacular variations of both the amplitude and shape of $\chi^{\prime}$ and $\chi^{\prime \prime}$ as frequency and temperature are changed. At the lowest frequencies and highest temperatures investigated (see Fig.1 and Fig.2a) the dissipationless $\chi^{\prime}(\varphi)$ is well described by a pure $2 \pi$-periodic cosine, as expected for the adiabatic susceptibility $\chi_{J}=\partial I_{J} / \partial \Phi$ of the Josephson current which is purely sinusoidal at these moderately high temperatures, much larger than $E_{T h}$ [4, 11]. As shown in Fig.1b,1c, The amplitude $\delta \chi_{\pi-0}^{\prime}=\chi^{\prime}(\pi)-\chi^{\prime}(0)$ perfectly reflects the expected, roughly exponential, decay of the Josephson critical current $I_{J}(T)=I_{J}(0) \exp \left(-k_{B} T / 3.6 E_{T h}\right)[\underline{8}$, that was also 


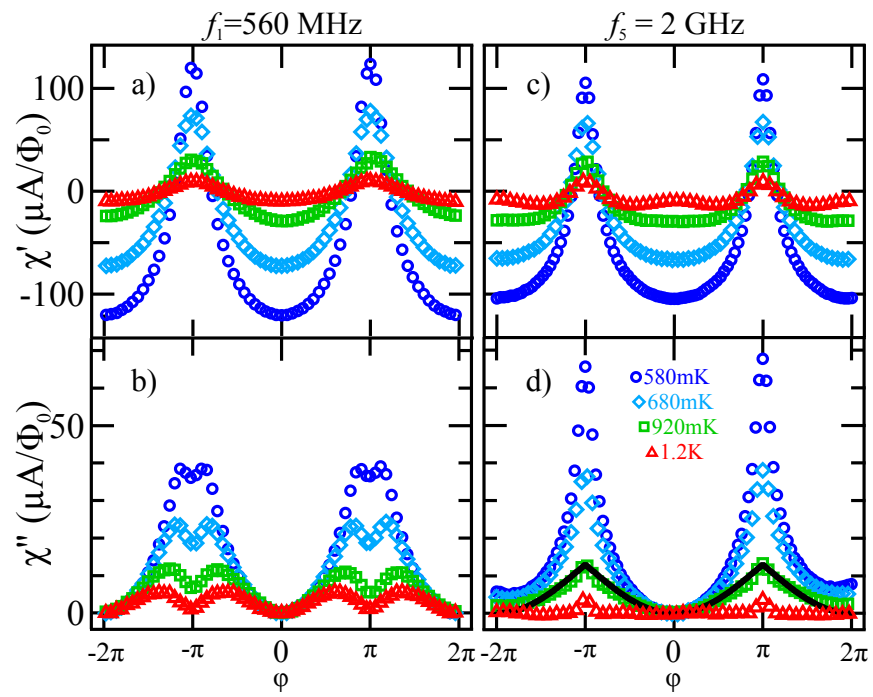

FIG. 2: Evolution of $\chi^{\prime}$ (top) and $\chi "$ (bottom) phase dependence with temperature at $f_{1}=560 \mathrm{MHz}<E_{T h} / h$ (left) and $f_{5}=2 G H z \gtrsim E_{T h} / h$ (right). $\chi^{\prime}(\varphi=\pi)$ and $\chi^{\prime \prime}(\varphi=\pi)$ increase with decreasing temperature. $\chi^{\prime}(\varphi)$ and $\chi^{\prime \prime}(\varphi)$ strongly differ at low frequency and high temperature whereas they are similar with a shape reminiscent of the minigap at high frequency. The solid line in $\mathrm{d}$ is proportional to the minigap dependence $|\cos (\varphi / 2)|$. Curves have been shifted so that $\chi^{\prime}(\varphi=\pi)-\chi^{\prime}(\varphi=0)=0$ and $\chi^{\prime \prime}(\varphi=0)=0$

measured in the control wire. We find for both samples $E_{T h}=71 \pm 5 \mathrm{mK}$ which corresponds to $\tau_{D} \simeq 0.1 \mathrm{~ns}$. In contrast, the dissipation, characterized by $\chi^{\prime \prime}(\varphi)$, is nearly $\pi$ periodic (see Fig.2b) at the largest temperatures investigated and acquires a strong $2 \pi$ periodic component at lower temperature. When increasing the frequency, (Fig.1b and Fig.2a and 2c ) $\chi^{\prime}(\varphi)$ contains additional harmonics, with peaks at odd multiples of $\pi$ and moreover a local maximum at $\varphi=0, \bmod [2 \pi]$ for the highest temperatures. On the other hand, at $2 \mathrm{GHz}$ (Fig2.c and 2.d ) and low temperature, $\chi^{\prime}(\varphi)$ and $\chi^{\prime \prime}(\varphi)$ have identical shapes, with peaks at $\pi$, mod $[2 \pi]$, reflecting the underlying minigap that varies like $|\cos (\varphi / 2)|$.

In the following we exploit this complex evolution of $\chi(\varphi)$ with frequency and temperature to extract the different mechanisms at work in the dynamics of Andreev states. To this end we make use of theoretical predictions [15] based on Usadel equations, and recent numerical simulations [19] inspired by the analysis of the ac response of normal mesocopic rings [20 22]. The response function of a NS ring has been shown to contain three contributions: $\chi=\chi_{J}+\chi_{D}+\chi_{N D}$. The adiabatic, zero frequency, Josephson contribution $\chi_{J}$ is purely real and is the derivative of the Josephson current $\partial I_{J} / \partial \Phi$. The second contribution, the diagonal susceptibility $\chi_{D}$, is the first non adiabatic, frequency dependent contribution. It describes the Debye-like relaxation of the (phase dependent) thermal populations $f_{n}(\varphi)$ of the Andreev states, with a typical inelastic relaxation time $\tau_{\text {in }}$ according to the simple model proposed for the dynamics of persistent currents in normal rings 2022 :

$$
\begin{aligned}
& \chi_{D}=\sum_{n} i_{n} \frac{\partial f_{n}}{\partial \Phi} \frac{i \omega}{1 / \tau_{i n}-i \omega}= \\
& -\sum_{n} i_{n}^{2} \frac{\partial f_{n}}{\partial \epsilon_{n}} \frac{i \omega}{1 / \tau_{i n}-i \omega},
\end{aligned}
$$

where the square of $i_{n}=-\partial \epsilon_{n} / \partial \Phi$, the current carried by the n-th Andreev level of energy $\epsilon_{n}(\Phi)$, appears. Finally, the non-diagonal contribution $\chi_{N D}$ describes quasi-resonant microwave-induced transitions between two Andreev levels, involving (in contrast with $\left.\chi_{D}\right)$ non diagonal matrix elements of the current operator [19]. The contribution $\chi_{N D}^{\prime \prime}$ to the phase dependent susceptibility dominates when $\omega \geq 1 / \tau_{D} \gg 1 / \tau_{i n}$, as in Fig.2d. $\chi^{\prime}$ and $\chi^{\prime \prime}$ then have similar shapes which follow approximately the minigap with peaks at $\pi$ and a $|\cos (\varphi / 2)|$ dependence[19]. This high frequency regime was the only one accessed in the previous experiments on $\mathrm{Au}$ wires directly connected to $\mathrm{W}$ superconducting wires. In those experiments the energy relaxation time, limited by electron electron interactions, of the order of $0.1 \mu \mathrm{s}$, was very long due to the superconducing contacts [12, 23]. Therefore those measurements were always in the regime $\omega \tau_{\text {in }} \gg 1$ where $\chi_{D}^{\prime \prime}$ is negligible, $(\mathrm{Eq} 2)$. In contrast, the $\mathrm{Pd}$ layer beneath the $\mathrm{Nb}$ contacts in the present samples considerably reduces the inelastic scattering time, leading to a substantial contribution of $\chi_{D}^{\prime \prime}$ for the resonator's first five eigenfrequencies. We now focus on this contribution analyzed in Fig.3 and Fig.4.

We first present the predicted flux dependence of $\chi_{D}$, given by the function $\mathrm{F}$

$$
F(\Phi, T)=\sum_{n} i_{n} \frac{\partial f_{n}}{\partial \Phi}=-\sum_{n} i_{n}^{2} \frac{\partial f_{n}}{\partial \epsilon_{n}},
$$

which reads in the continuous spectrum limit $F(\Phi, T)=$ $\int d \epsilon J_{S}^{2}(\Phi, \epsilon) /\left[4 k_{B} T \rho(\epsilon) \cosh ^{2}\left(\epsilon / 2 k_{B} T\right)\right]$. Here $J_{S}$ and $\rho$ are respectively the spectral current and the density of states of the SNS junction. This function was introduced by Lempitsky [1] to describe the I(V) characteristics of SNS junctions, and was calculated numerically using Usadel equations by Virtanen et al.15. At large temperature compared to $E_{T h}, F(\Phi, T)$ can be approximated by the following analytical form: $F_{U}(\varphi, T) \propto$ $[(-\pi+(\pi+\varphi)[2 \pi])] \sin (\varphi)-|\sin (\varphi)| \sin ^{2}(\varphi / 2) / \pi$. It is dominated by its second harmonics with in addition a sharp linear singularity at odd multiples of $\pi$ (see Fig.4). This is due to the dominant contribution of Andreev levels close to the minigap whose flux dependence is singular like in a highly transmitting superconducting single channel point contact [2]. We now show that the particular flux dependence of the function $F_{U}$ can explain the experimental data of Fig.1 and 2. We first follow the frequency dependence of the amplitude of $\chi_{D}^{\prime}(\varphi)=\chi^{\prime}(\varphi)-\chi_{J}(\varphi)$ at fixed temperature, and check that the shape of $\chi_{D}^{\prime}(\varphi)$ 

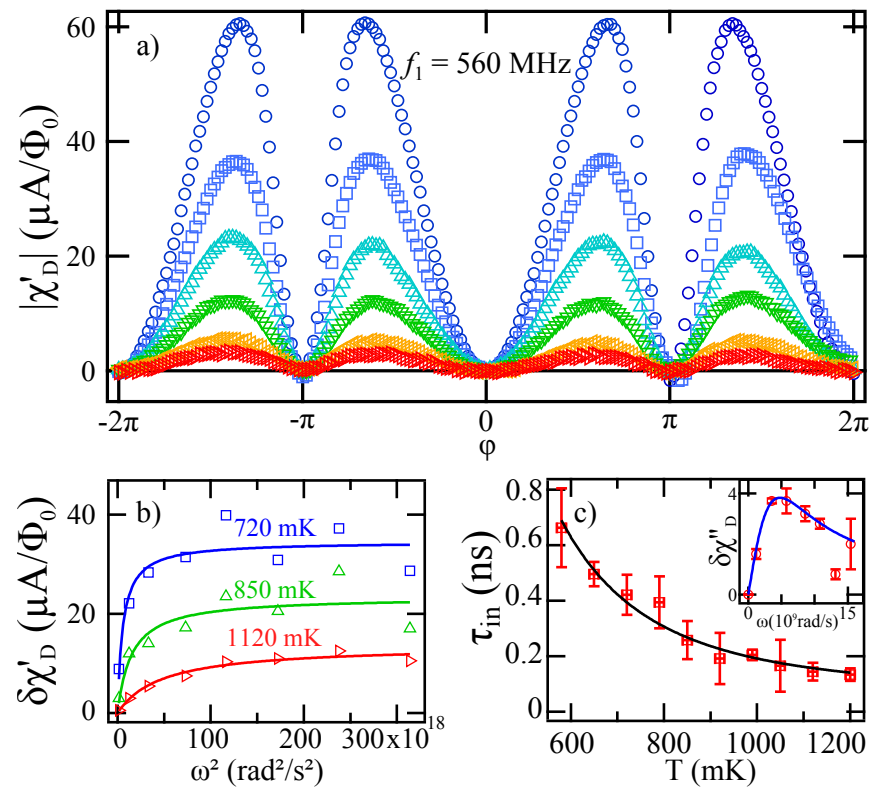

FIG. 3: a. Experimental phase dependence of $\chi_{D}^{\prime}$ at $f_{1}=560 \mathrm{MHz}$ and (from top to bottom) $T=580 \mathrm{mK}$, $650 m K, 720 m K, 820 m K, 1000 m K, 1200 m K$. In agreement with theory [15], the position of the maximum slightly shifts to lower values with decreasing temperature. b. Frequency dependence of $\delta \chi_{D}^{\prime}$ : the maximum of $-\chi_{D}^{\prime}(\varphi)$, at different temperatures (triangles) compared to the theoretical prediction from Eq.3. c. Temperature dependence of extracted $\tau_{i n}$ (see text). Solid line is a $T^{-3}$ law. Inset: frequency dependence of $\delta \chi_{D}^{\prime \prime}$ at $1.15 \mathrm{~K}$ (circles) compared to the theoretical prediction from Eq.3.

does not change with frequency and is the same as that of $\chi_{D}^{\prime \prime}$, as predicted for the temperature and frequency regime where the contribution of $\chi_{N D}^{\prime \prime}$ can be neglected. As shown on Fig.3b it is then possible to fit the frequency dependence of the amplitude of $\chi_{D}^{\prime}(\varphi)$ by the expected $\left(\omega \tau_{i n}\right)^{2} /\left(1+\left(\omega \tau_{i n}\right)^{2}\right)$ and determine the characteristic time $\tau_{i n}$ for several temperatures according to Eq2. We find values of $\tau_{i n}$ varying between 0.2 and $1 \mathrm{~ns}$, quite similar to what was deduced from weak localisation measurements in Pd thin films [16. Moreover the power law decrease of $\tau_{i n}(T)$ in $T^{-3}$, is in reasonable agreement with what is expected for paramagnons which constitute the dominant inelastic scattering at low temperature in $\mathrm{Pd}$ which is close to a ferromagnetic transition. It is also interesting to note that our results can be described by a single inelastic time, independent of $\varphi$, whereas a phase dependent $\tau_{i n}$ is expected for electron phonon collisions in SNS junctions 24]. This is probably due to the fact that temperature is larger than $E_{g}(0)$ in our case.

A similar analysis can be done on $\chi^{\prime \prime}$, the quality of the calibration is however not as good as on $\chi^{\prime}$. Moreover we still lack a good analytical prediction for $\chi_{N D}(\varphi)$, which gives a large contribution to $\chi^{\prime \prime}(\varphi)$ at low temperature and high frequency. We have overcome this difficulty by subtracting for frequencies larger than $1.7 \mathrm{GHz}$ the flux

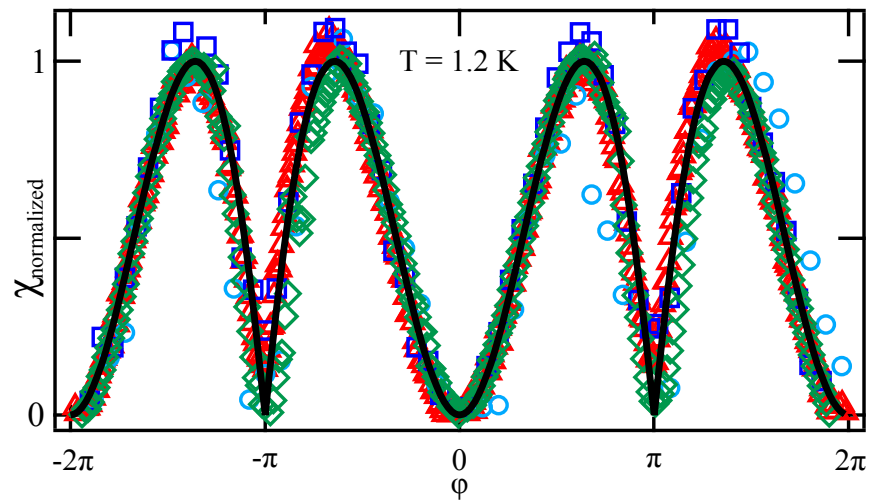

FIG. 4: Normalized experimental phase dependences at $T=1.2 \mathrm{~K}$ (open symbols) compared to $F_{U}$ (solid). Data are $\chi "$ (squares), $\left|\chi_{D}^{\prime}\right|=\chi_{J}-\chi^{\prime}$ (circles) at $f_{1}=560 \mathrm{MHz}$ and $\chi "$ (triangles), $\left|\chi_{D}^{\prime}\right|=\chi_{J}-\chi^{\prime}$ (diamonds) at $f_{3}=1.35 \mathrm{GHz}$. At high temperature and for $f \lesssim E_{T h} / h$, experimental $\chi_{J}-\chi^{\prime}$ and $\chi$ " are found to be in very good agreement with theoretical prediction of Usadel equations $F_{U}$.

dependence of $\chi_{N D}^{\prime \prime}$ estimated from the high frequency data $(2.8 \mathrm{GHz})$. The resulting amplitude $\delta \chi_{D}^{\prime \prime}(\omega)$ agrees with the expected frequency dependence in $\omega \tau_{i n} /(1+$ $\left.\left(\omega \tau_{i n}\right)^{2}\right)$ as shown in the inset of Fig.3c. One can also compare the independently measured flux dependences of $\chi^{\prime}-\chi_{J}$ and $\chi^{\prime \prime}$ with theoretical predictions from the Usadel equations, $F_{U}(\Phi, T)$. This is done in Fig.4 for several frequencies and a good agreement is found.

With this set of experiments, we have thus shown that the frequency and temperature dependences of the response function of NS rings in a time dependent flux are consistent with a simple Debye relaxation model of the population of the Andreev levels. Using fluctuation dissipation theorem one can estimate the related thermodynamic current noise as:

$S_{I}(\omega)=\frac{2}{\pi} \frac{k_{B} T \chi_{D}^{\prime \prime}(\omega)}{\omega}=\frac{2}{\pi} k_{B} T \sum_{n} i_{n}^{2}(\varphi) \frac{\partial f_{n}}{\partial \epsilon_{n}}\left[\frac{\tau_{\text {in }}}{1+\left(\omega \tau_{i n}\right)^{2}}\right]$.

The measurement of the ac current linear response of an NS ring to an ac flux thus reveals two fundamental mechanisms contributing to dissipation at finite frequency. One of them, predominant at high frequency and low temperature, describes the physics of microwave induced transitions above the minigap. We have clearly identified and characterized the second cause of dissipation, the thermal relaxation of the populations of the Andreev states. It is described by an inelastic rate which is extremely sensitive to the nature of the NS interface. This dissipative response is directly related to the low frequency thermal noise of the Josephson current, with a flux dependence proportional to the average square of the spectral (or single level) current, and can be precisely described by theoretical predictions. These results show that linear ac measurements in a wide range of fre- 
quency, close to equilibrium, reveal physical properties of SNS junctions that are not accessible by standard transport measurements dominated by non linear effects. The type of experiments presented here is uniquely suited to investigate more exotic systems, for instance with the normal diffusive wire replaced by a ballistic wire, leading to a discrete Andreev spectrum known to be extremely sensitive to spin orbit interactions.

We acknowledge A. Kasumov and F. Fortuna for help with the FIB and M. Aprili, F. Chiodi, R. Deblock, M. Feigelman, T.T. Heikkilä, K. Tikhonov and P. Virtanen for fruitful discussions.

[1] S.V. Lempitsky Sov. Phys. JETP 57, 910(1983).

[2] D. Averin and H. T. Imam Phys. Rev. Lett. 76, 3814 (1996). A. Martin-Rodero and A. Levy Yeyati, F. J. Garcy a-Vidal Phys.Rev.B 53, R8891 (1996).

[3] Kulik, I., Sov. Phys. JETP 30, 944 (1970).

[4] T. T. Heikkilä, J. Särkkä, and F. K. Wilhelm Phys. Rev.B66, 184513 (2002).

[5] F. Zhou et al., J. Low Temp. Phys. 110, 841 (1998).

[6] Dmitri A. Ivanov, Raphael von Roten, and Gianni Blatter Phys. Rev. B66, 052507 (2002).

[7] K.W. Lehnert et al. Phys. Rev. Lett. 82, 1265 (1999).

[8] Dubos, P. et al., Phys. Rev. B 63, 064502 (2001). L. Angers, F. Chiodi, J. C. Cuevas, G. Montambaux, M. Ferrier, S. Gueron, H. Bouchiat Phys. Rev B77, 165408 (2008).

[9] H. le Sueur et al., Phys. Rev. Lett. 100, 197002 (2008).

[10] A. A. Golubov, M. Yu. Kupriyanov, and E. Illichev Rev.
Mod. Phys. 76, 411 (2004).

[11] M. Fuechsle et al., Phys. Rev. Lett. 102, 127001 (2009).

[12] F. Chiodi et al, Sci. Rep,1 (2011).

[13] D. Rogovin and D. J. Scalapino, Ann. Phys. (N.Y.) 86, 1 (1974).

[14] I. O. Kulik and A. N. Omelyanchuk, Sov. J. Low Temp.Phys. 3, 459 (1977).

[15] Virtanen, P., Cuevas, J.C., Bergeret, F.S., and Heikkilä, Phys. Rev. B 83, 144514 (2011).

[16] H. Raffy et al J. Physique 46 627,(1985).

[17] L. Dumoulin, P. Nedellec, and P. M. Chaikin, Phys. Rev. Lett. 47, 208 (1981).

[18] M. Wolz, C. Debuschewitz, W. Belzig, and E. Scheer Phys. Rev.B84, 104516 (2011).

[19] B. Dassonneville, M. Ferrier, S.Guéron and H.Bouchiat (unpublished)

[20] M. Büttiker, Annals of the New York Academy of Sciences,480, 194 (1986).

[21] N. Trivedi and D.A. Browne, Phys. Rev. B 38(14), 9581 (1988)

[22] B. Reulet and H. Bouchiat, Phys. Rev. B 50(4), 2259 (1994), A. Kamenev, B. Reulet, H. Bouchiat and Y. Gefen, Europhys. Lett. 28 (6), 391 (1994).

[23] Y. Blanter Phys. Rev. B54 12807, (1997), C. Texier and G. Montambaux Phys. Rev. B72, 115327 (2005). It is shown that the electron-electron scattering time varies like $g h / k T$ in finite size quasi $1 \mathrm{D}$ wires instead of the $T^{-2 / 3}$ dependence observed for infinitely long wires.

[24] T. Heikkila and F. Giazotto Phys. Rev. B 79, 094514 (2009)

[25] A. Buzdin, Phys. Rev. Lett. 101, 107005 (2008), D. Sau, R. M. Lutchyn, S. Tewari, S. Das Sarma, Phys. Rev. Lett. 104, 040502 (2010), M.Cheng and R. M. Lutchyn Phys. Rev. B86, 134522 (2012). 